\title{
Probiotic and prebiotic use by patients with inflammatory bowel disease
}

\author{
C. R. H. Hedin ${ }^{1}$, M. Graczer ${ }^{1}$, J. D. Sanderson ${ }^{1}$, J. O. Lindsay ${ }^{2}$ and K. Whelan ${ }^{1}$ \\ ${ }^{1}$ Nutritional Sciences Division, King's College London, London SE1 9NN, UK and ${ }^{2}$ Barts and the London NHS Trust, \\ London E1 1BB, UK
}

The gastrointestinal microbiota are essential in inflammatory bowel disease (IBD) pathogenesis. Consequently, investigation into the use of probiotics and prebiotics to manipulate the microbiota has flourished. The evidence supporting their use, although frequently contradictory, is strongest for the treatment of ulcerative colitis (UC) ${ }^{(1)}$. The aim of the present study was to investigate the current use of probiotics and prebiotics in patients with IBD.

Adults with Crohn's disease (CD) or UC attending IBD clinics at Bart's and the London National Health Service (NHS) Trust and Guy's and St Thomas' NHS Foundation Trust underwent face-to-face interviews about their probiotic and prebiotic use. Demographic, clinical (e.g. disease type, drug dose), health-related behaviour (e.g. smoking) and psychology (e.g. disease-specific multidimensional health locus of control) data were collected. Knowledge of probiotics and prebiotics was measured by scoring patients' definitions of each on a pre-defined scale. Continuous data were compared using an independent $t$ test. Categorical data were compared using a $\chi^{(2)}$ or Fisher's exact test, as appropriate.

A total of 136 patients were interviewed (eighty-two (60\%) with CD; fifty-four (40\%) with UC). Only four (3\%) patients had ever used prebiotics for their IBD and none of these patients was a current user. In contrast, sixty (44\%) patients had ever used probiotics for their IBD and thirty $(22 \%)$ were current users. Significantly more patients with UC $(n 31 ; 57 \%)$ than with CD $(n 29 ; 35 \%)$ had ever used probiotics for their IBD $(P=0.011)$. Previous probiotic use was higher in Christians $(P=0.039)$, non-smokers $(P=0.045)$ and when disease duration was $<5$ years $(P=0.025) v$. respective comparison groups. Locus of control was not associated with probiotic use. In total, fifty-eight (42\%) and $132(97 \%)$ patients were unable to give a definition for probiotics and prebiotics, respectively. However, users of probiotics had significantly higher knowledge scores (mean 1.5 (SD 1.0)) than non-users (mean 0.6 (SD 0.8); $P=0.043$ ). Information about probiotics and prebiotics was gained from friends or family $(23 \%)$, doctors or gastroenterologists $(21 \%)$ and television $(18 \%)$. Of the probiotic or prebiotic users only thirty-three $(54 \%)$ had discussed their use with a healthcare professional.

The present study has identified a high rate of use of probiotics and prebiotics in patients with IBD. Patients choosing to take probiotics were those most likely to benefit (i.e. those with UC), possibly reflecting the penetration of knowledge of the clinical evidence. However, although better informed than non-users, many patients adopting this therapy had limited knowledge, perhaps reflecting their reliance on friends, family and television for information. Often, healthcare teams were unaware of their patients' use of probiotics and prebiotics, precluding integrated prescribing and care. Uptake of these treatments somewhat surpasses the evidence of their effectiveness. It is therefore essential that healthcare teams address these therapies in partnership with patients to effectively advise them and facilitate integrated care.

1. Hedin CRH, Whelan K \& Lindsay JO (2007) Proc Nutr Soc 66, 307-315.

2. Wallston, Stein \& Smith (1994) J Personality Assessment 63, 534-553. 